\title{
The First Total Synthesis of the Lipid Mediator PD2n-3 DPA
}

\author{
Jørn Eivind Tungen, ${ }^{\dagger}+$ Karoline Gangestad Primdahl, ${ }^{\dagger},+,{ }^{*}$ and Trond Vidar Hansen ${ }^{\dagger}$ \\ 'Department of Pharmacy, Section for Pharmaceutical Chemistry, University of Oslo, P.O. \\ Box 1068, 0316 Oslo, Norway
}

\section{General information}

Unless otherwise stated, all commercially available reagents and solvents were used in the form they were supplied without any further purification. The stated yields are based on isolated material. All reactions were performed under an argon atmosphere using Schlenk techniques. Reaction flasks were covered with aluminium foil during reactions and storage to minimize exposure to light. Thin layer chromatography was performed on silica gel 60 F254 aluminumbacked plates fabricated by Merck. Flash column chromatography was performed on silica gel $60(40-63 \mu \mathrm{m})$ produced by Merck. NMR spectra were recorded on a Bruker AVII600, Bruker AVII400 or a Bruker DPX300 spectrometer at $600 \mathrm{MHz}, 400 \mathrm{MHz}$ or $300 \mathrm{MHz}$ respectively for ${ }^{1} \mathrm{H}$ NMR and at $150 \mathrm{MHz}, 100 \mathrm{MHz}$ or $75 \mathrm{MHz}$ respectively for ${ }^{13} \mathrm{C}$ NMR. Coupling constants $(J)$ are reported in hertz and chemical shifts are reported in parts per million $(\delta)$ relative to the central residual protium solvent resonance in ${ }^{1} \mathrm{H} \mathrm{NMR}\left(\mathrm{CDCl}_{3}=\delta 7.26\right.$, DMSO$d_{6}=\delta 2.50$ and MeOD- $\left.d_{4}=\delta 3.31\right)$ and the central carbon solvent resonance in ${ }^{13} \mathrm{C}$ NMR $\left(\mathrm{CDCl}_{3}=\delta 77.00 \mathrm{ppm}, \mathrm{DMSO}-d_{6}=\delta 39.43\right.$ and MeOD$\left.-d_{4}=\delta 49.00\right)$. Optical rotations were measured using a $1 \mathrm{~mL}$ cell with a $1.0 \mathrm{dm}$ path length on a Perkin Elmer 341 polarimeter. Mass spectra were recorded at $70 \mathrm{eV}$ on Micromass Prospec Q or Micromass QTOF $2 \mathrm{~W}$ spectrometer using ESI as the method of ionization. High-resolution mass spectra were recorded at $70 \mathrm{eV}$ on Micromass Prospec Q or Micromass QTOF 2W spectrometer using ESI as the method of ionization. HPLC-analyses were performed using a $\mathrm{C} 18$ stationary phase (Eclipse XDB-C18, 4.6 × $250 \mathrm{~mm}$, particle size $5 \mu \mathrm{m}$, from Agilent Technologies), applying the conditions stated. The UV/Vis spectrum was recorded using an Agilent Technologies Cary 8485 UV-VIS spectrophotometer using quartz cuvettes. Separation of isomers were conducted using Biotage ${ }^{\circledR}$ Select purification system (Biotage ${ }^{\circledR}$ Sfär-C-18) applying the conditions stated. 


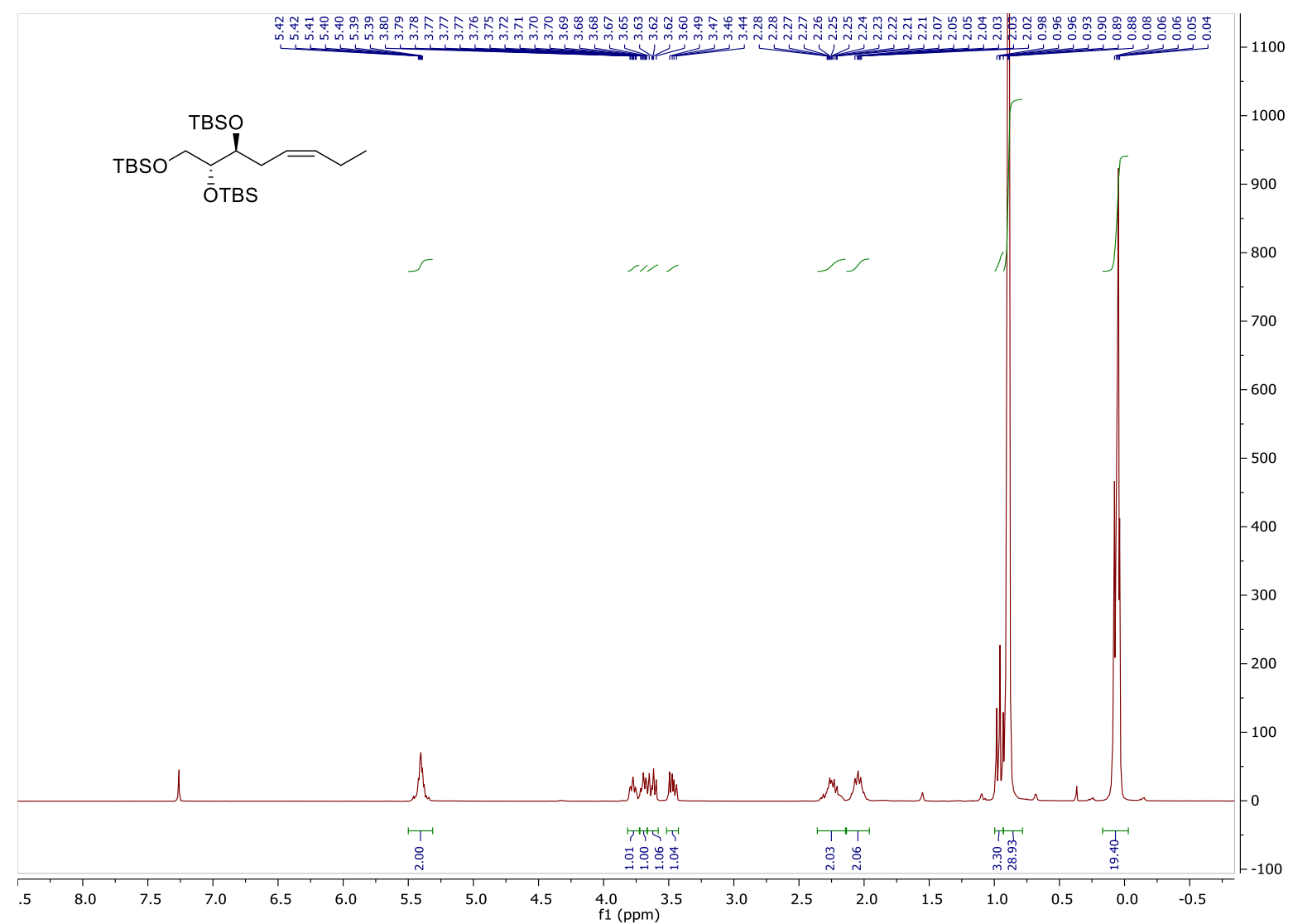

Figure S-1 ${ }^{1} \mathrm{H}$-NMR spectrum of compound 16.

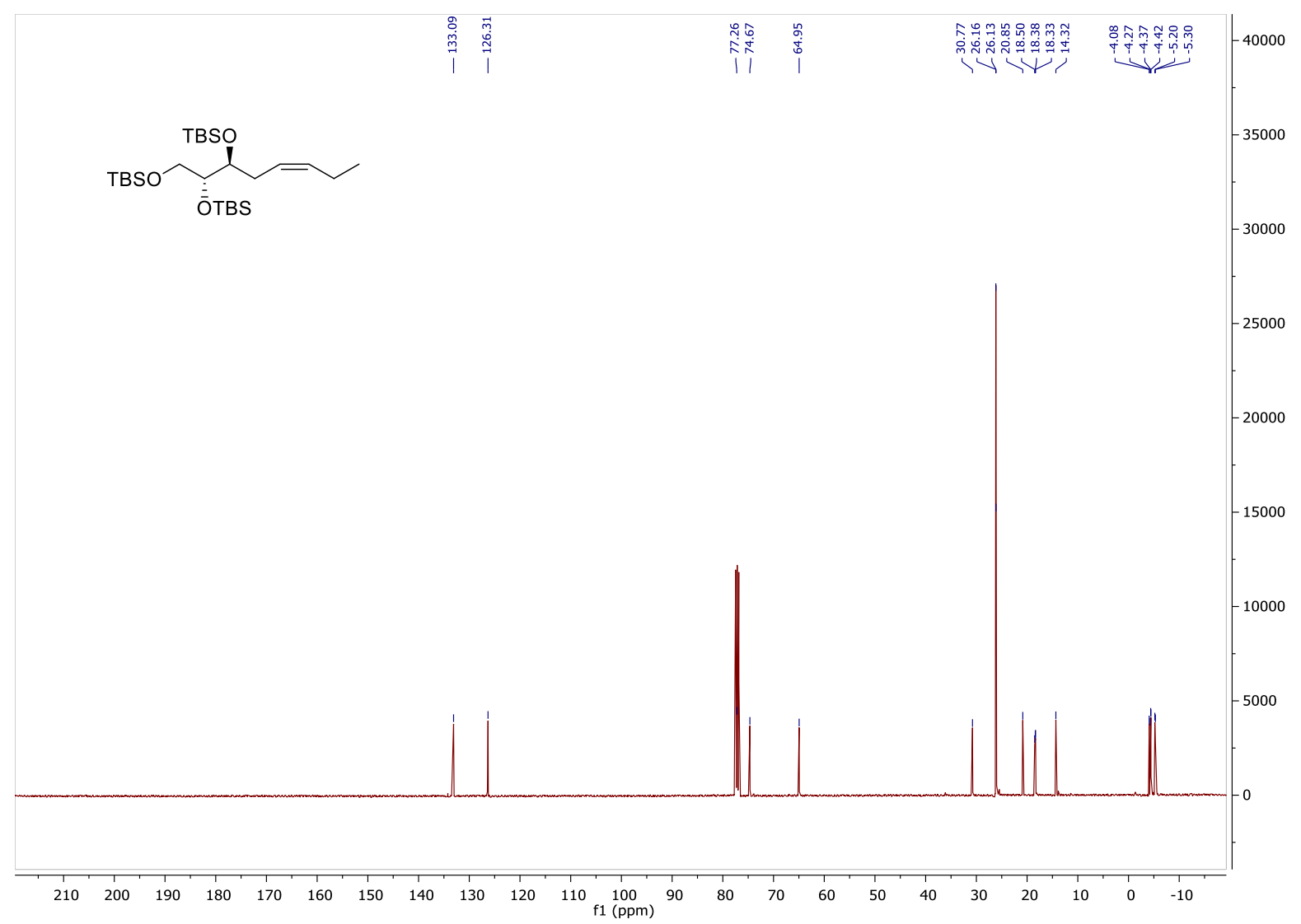

Figure S-2 ${ }^{13} \mathrm{C}-\mathrm{NMR}$ spectrum of compound $\mathbf{1 6}$. 


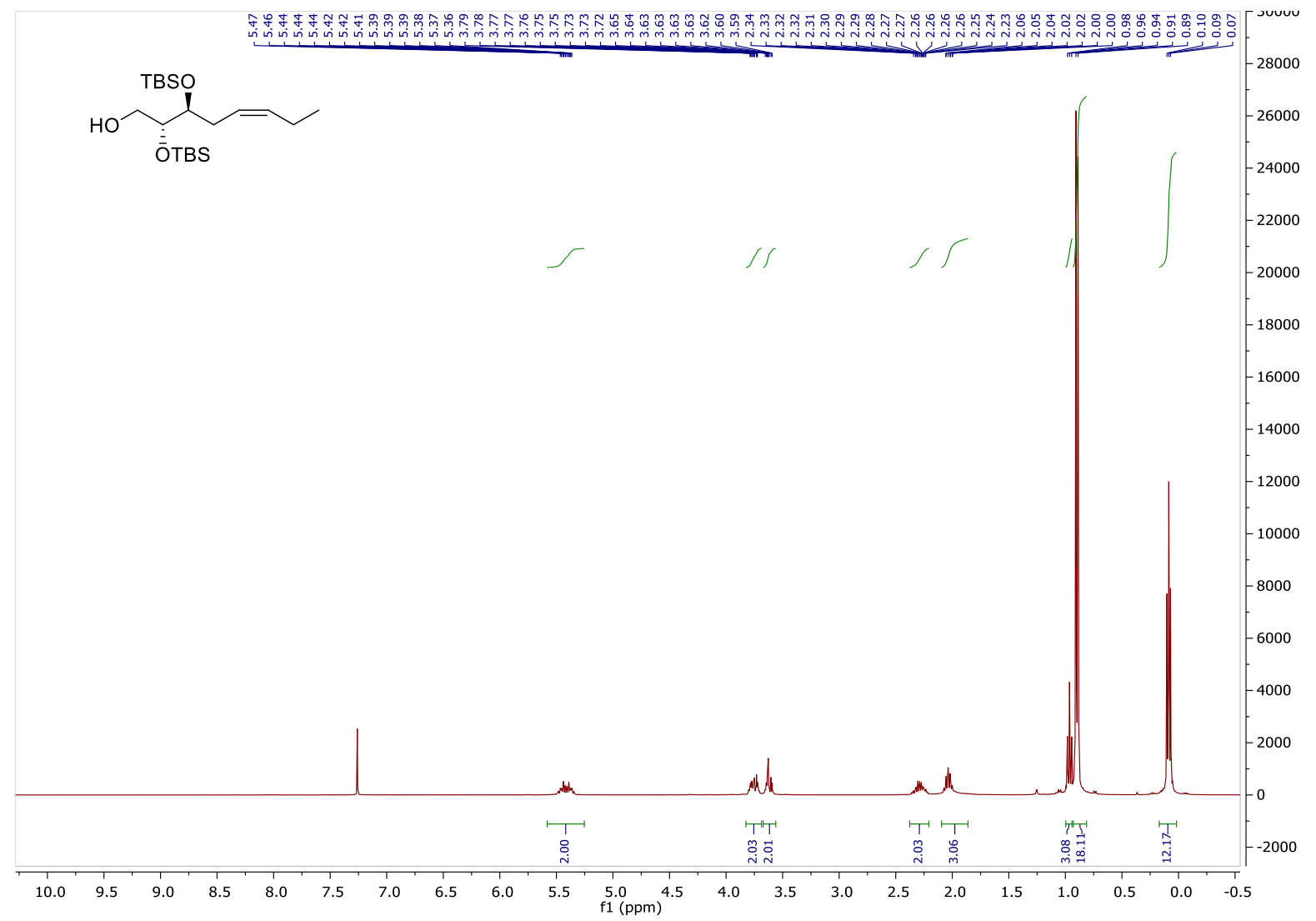

Figure S-3 ${ }^{1} \mathrm{H}-\mathrm{NMR}$ spectrum of compound 17 .

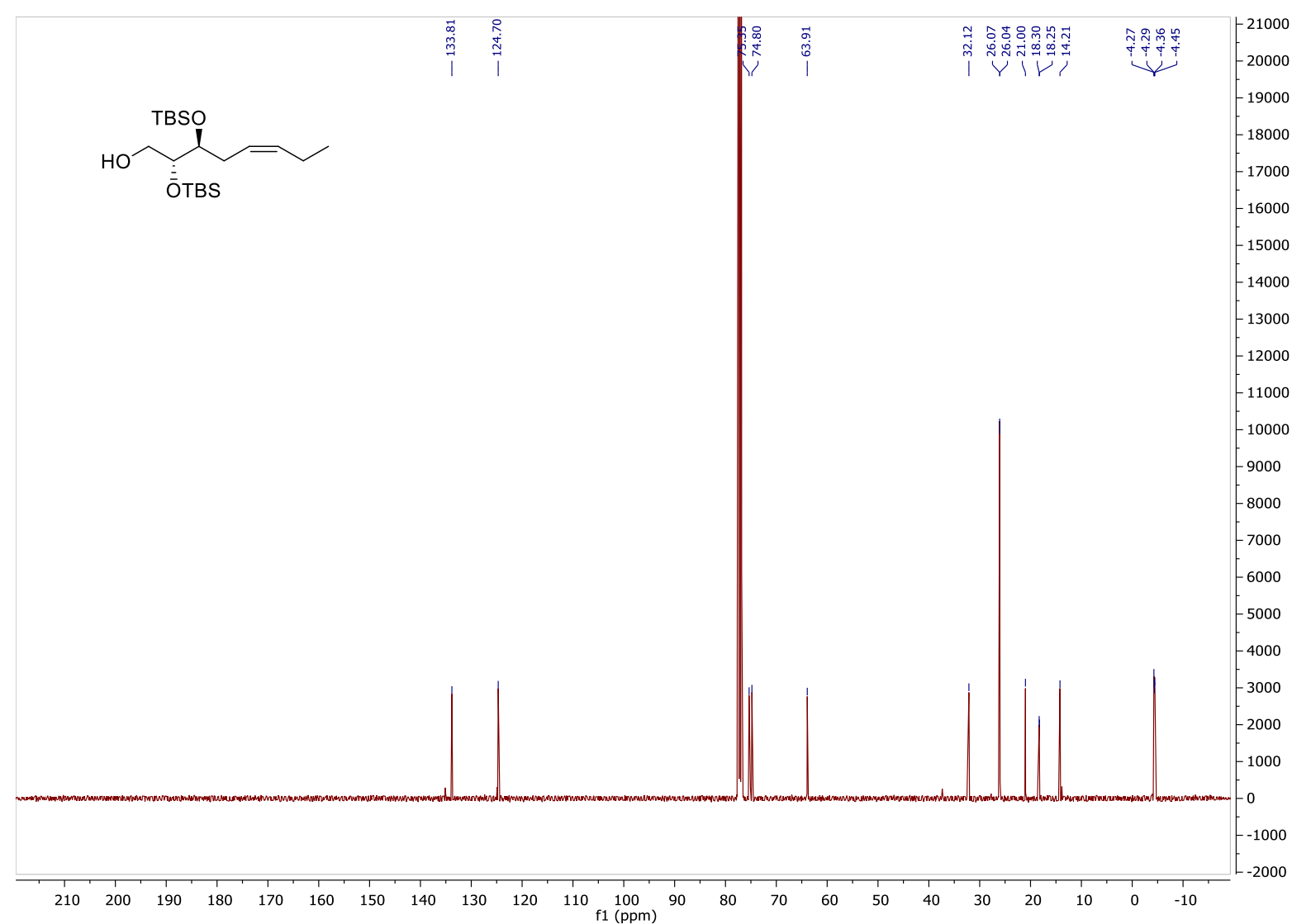

Figure S-4 ${ }^{13}$ C-NMR spectrum of compound 17 . 


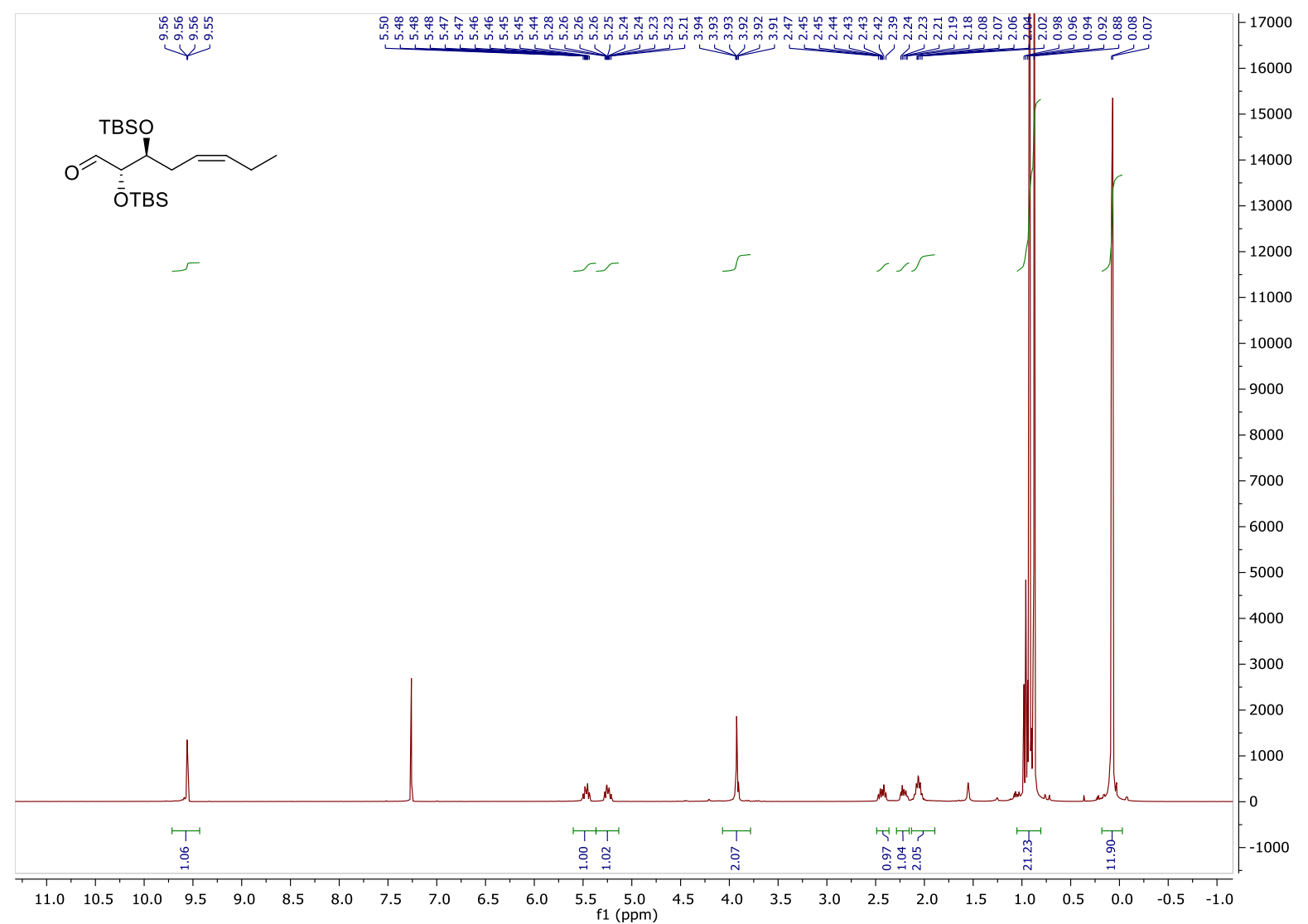

Figure S-5 ${ }^{1}$ H-NMR spectrum of compound 9.

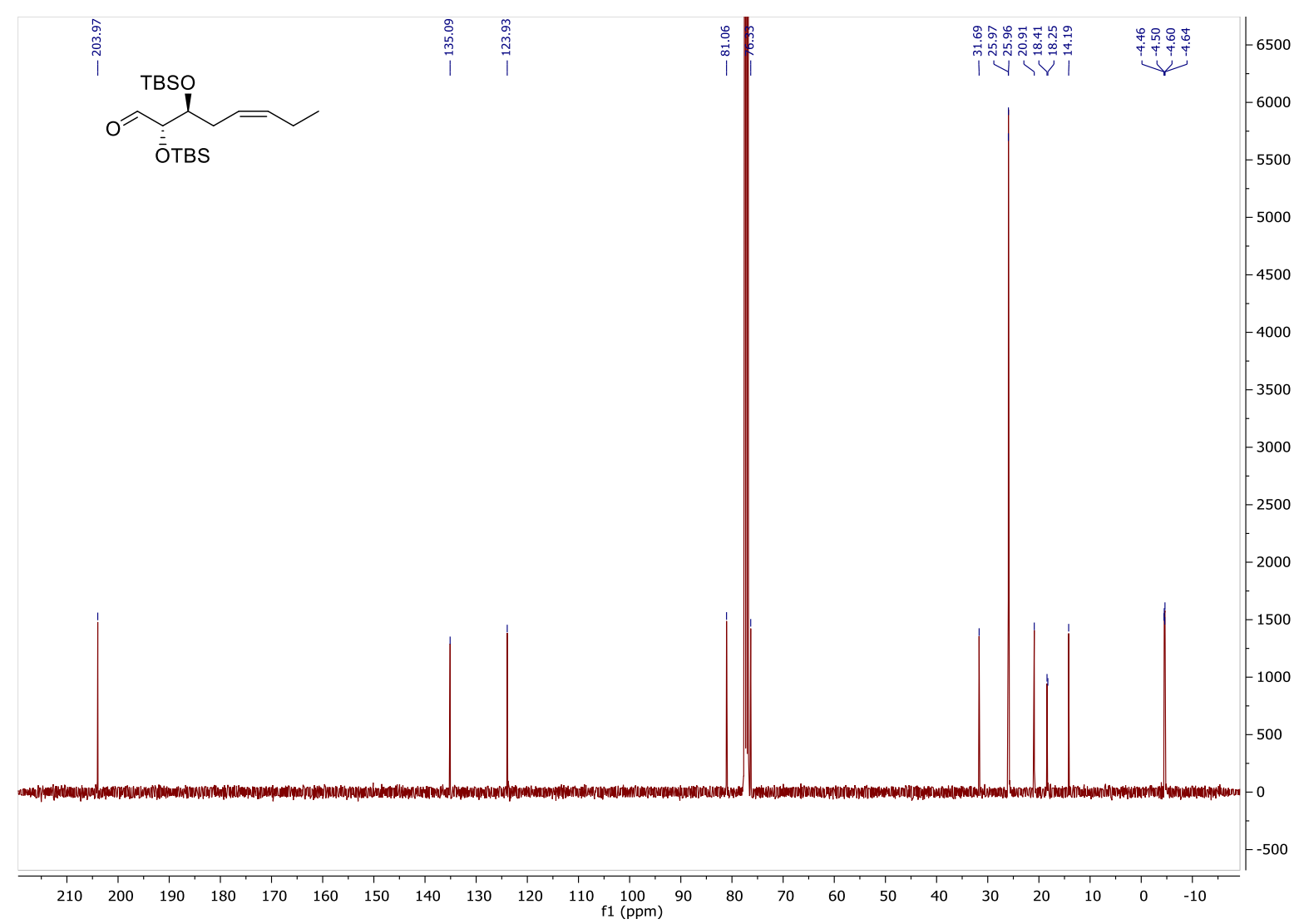

Figure S-6 ${ }^{13} \mathrm{C}$-NMR spectrum of compound 9 . 


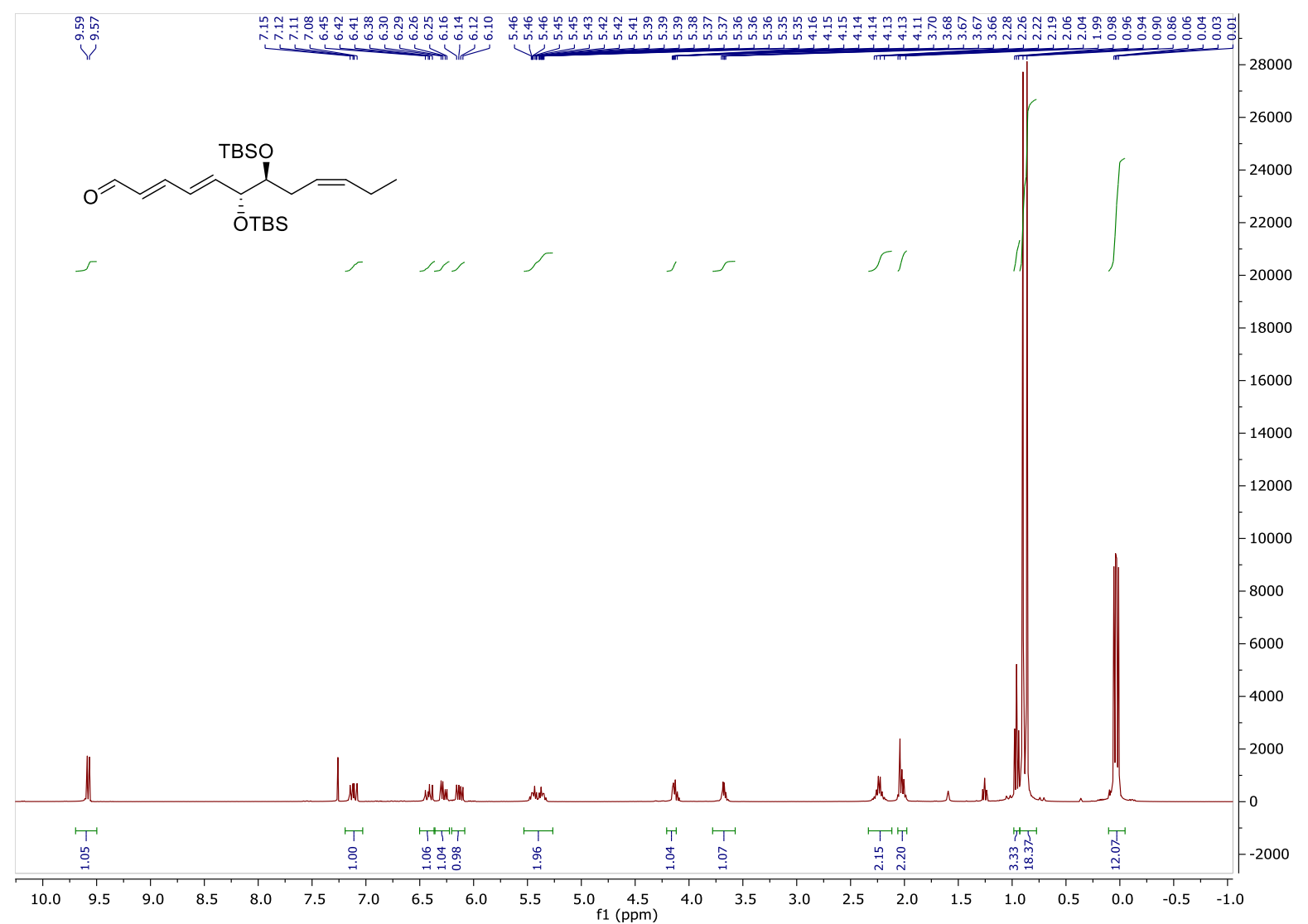

Figure S-7 ${ }^{1} \mathrm{H}-\mathrm{NMR}$ spectrum of compound 7.

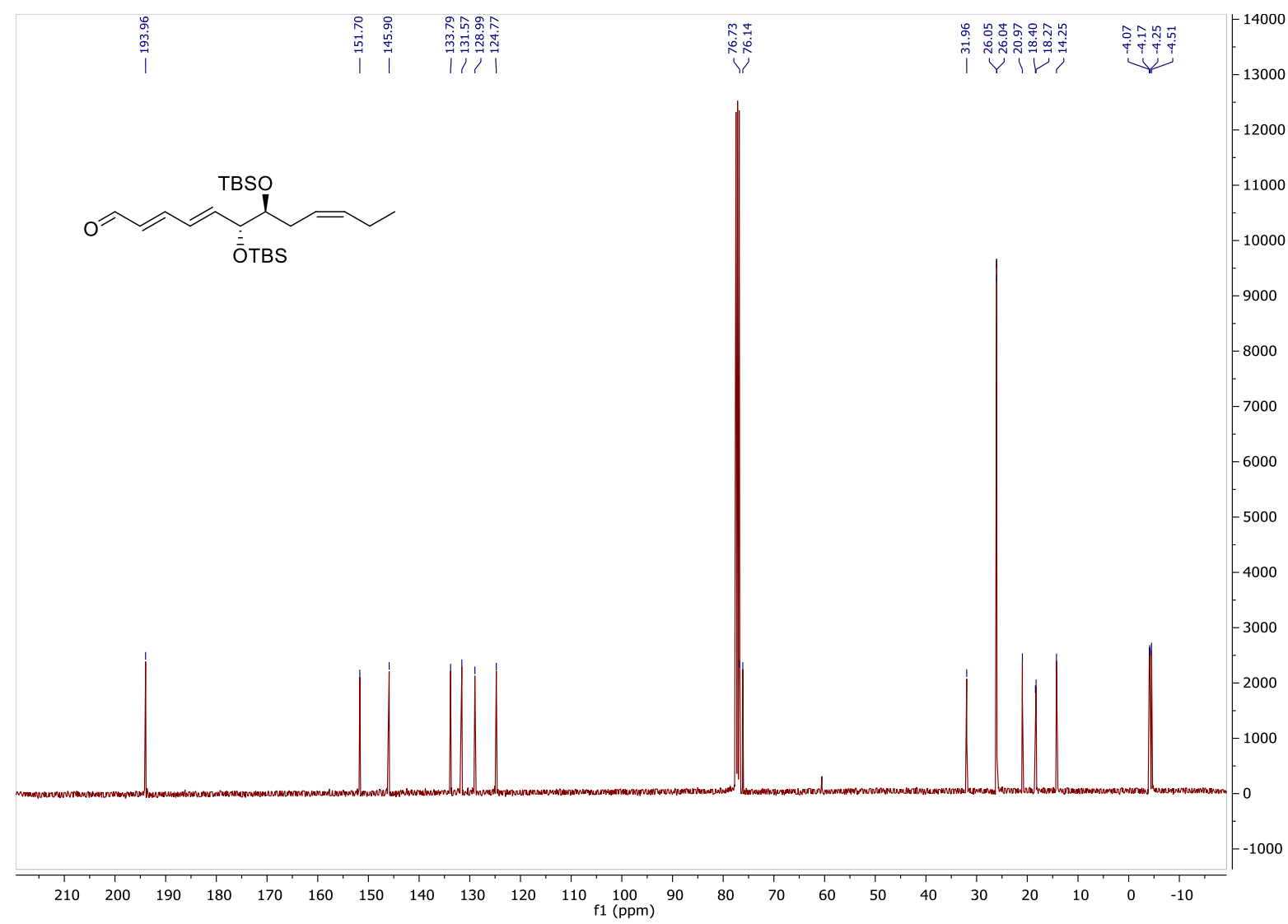

Figure S-8 ${ }^{13} \mathrm{C}-\mathrm{NMR}$ spectrum of compound 7 . 


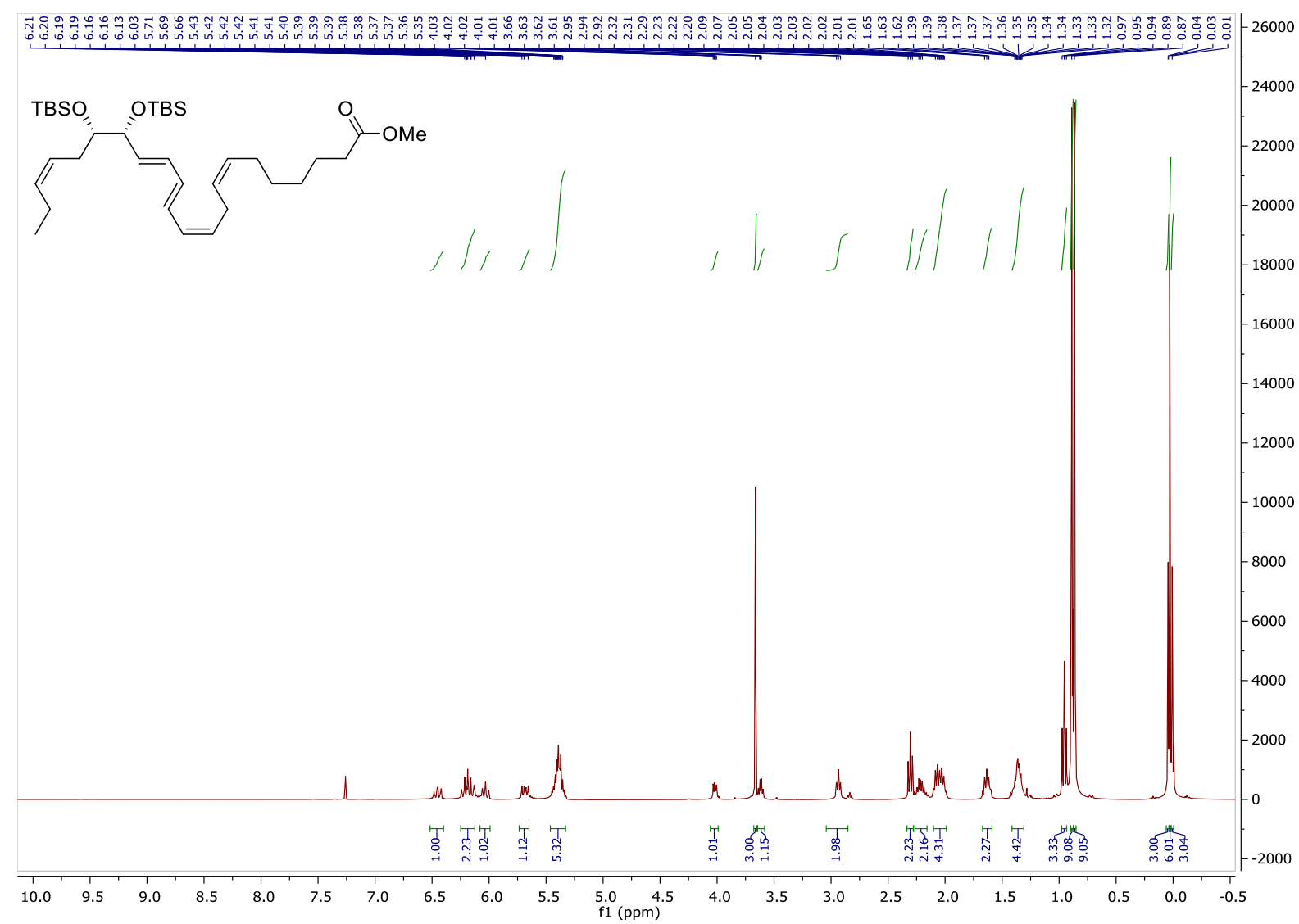

Figure S-9 ${ }^{1} \mathrm{H}-\mathrm{NMR}$ spectrum of compound 20.

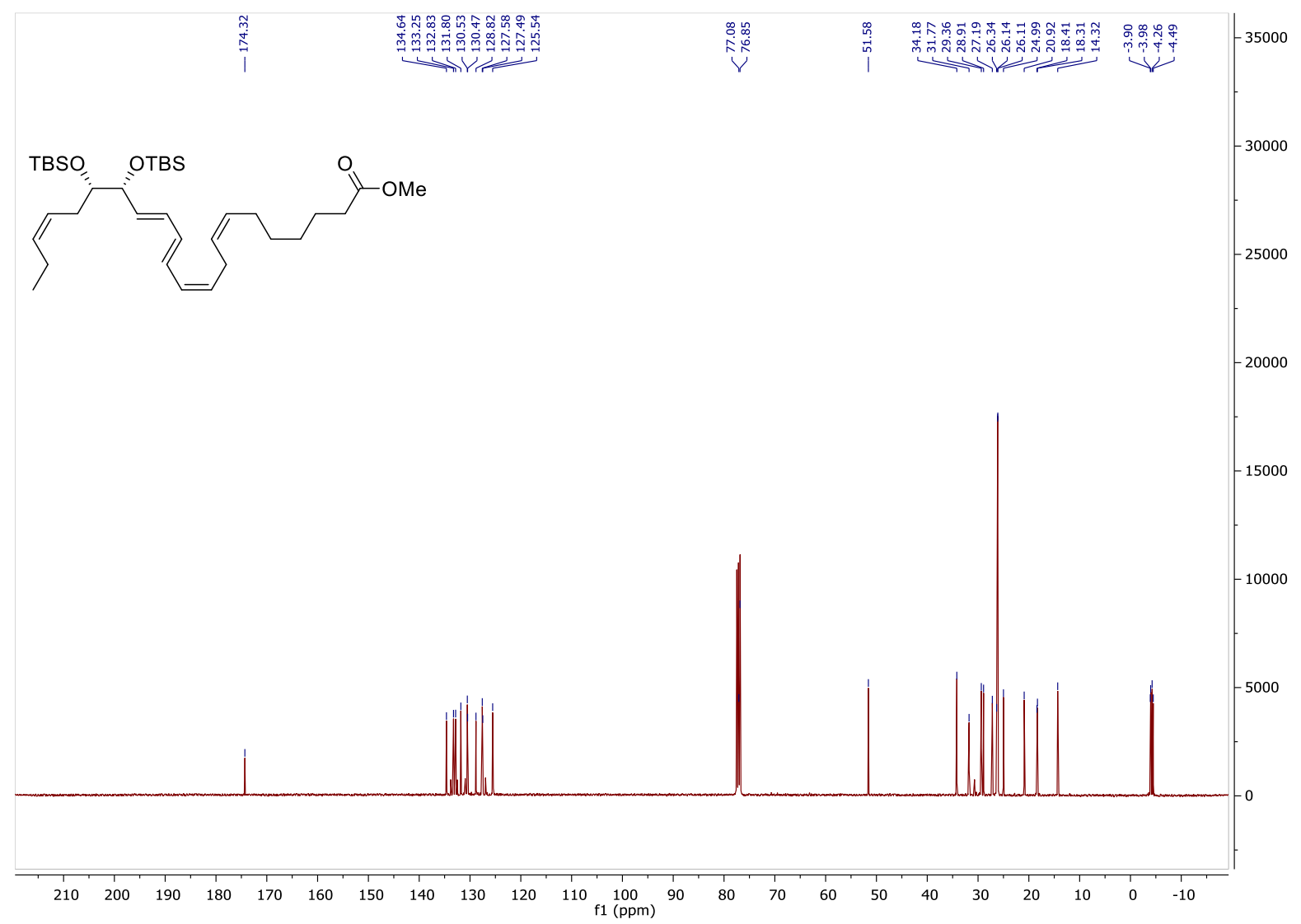

Figure S-10 ${ }^{13} \mathrm{C}$-NMR spectrum of compound 20. 


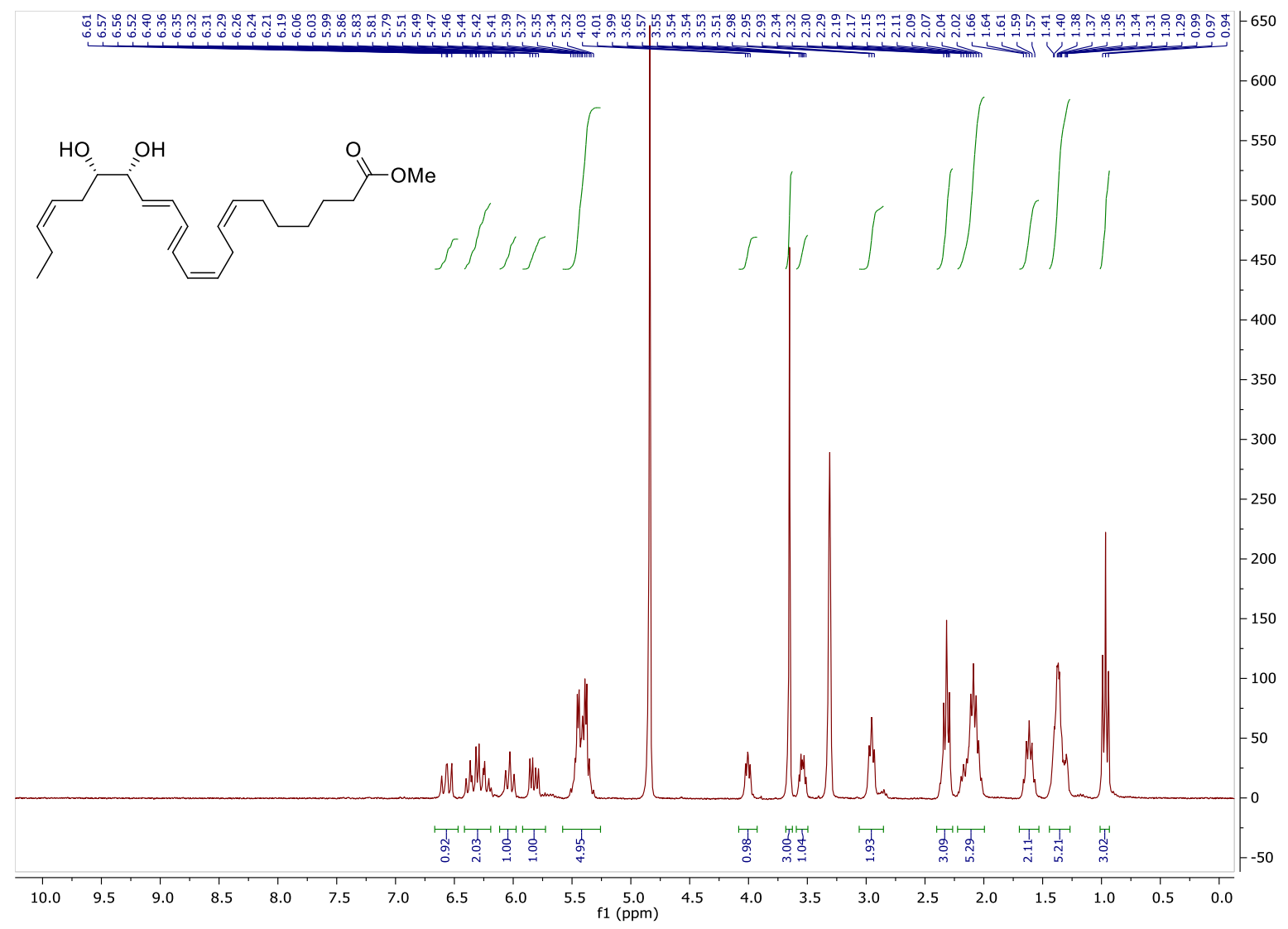

Figure S-11 ${ }^{1} \mathrm{H}-\mathrm{NMR}$ spectrum of the methyl ester of PD $2_{\mathrm{n}-3 \mathrm{DPA}}(\mathbf{2 1})$.

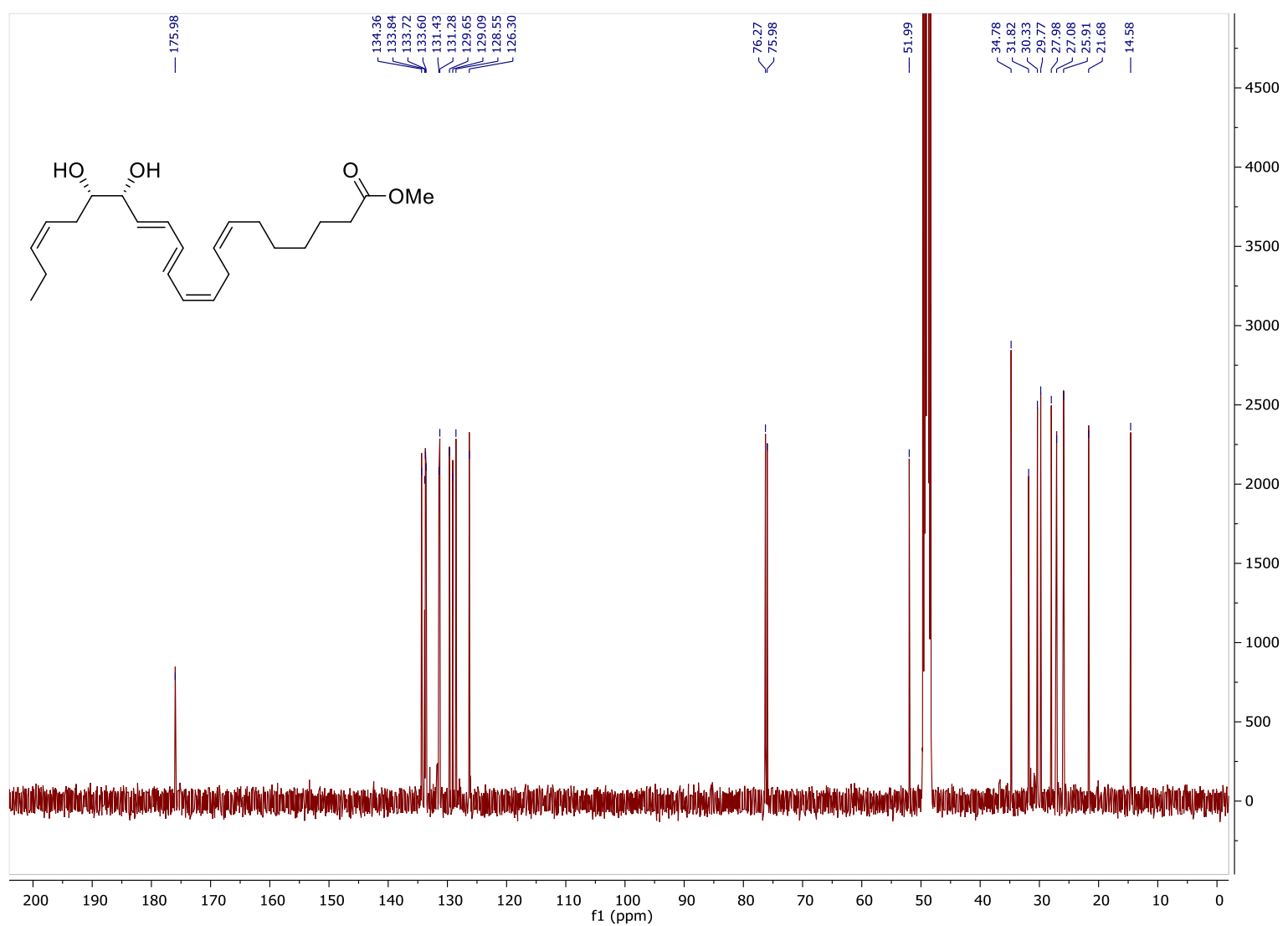

Figure S-12 ${ }^{13} \mathrm{C}-\mathrm{NMR}$ spectrum of the methyl ester of PD2 $2_{\mathrm{n}-3 \mathrm{DPA}}(\mathbf{2 1})$. 


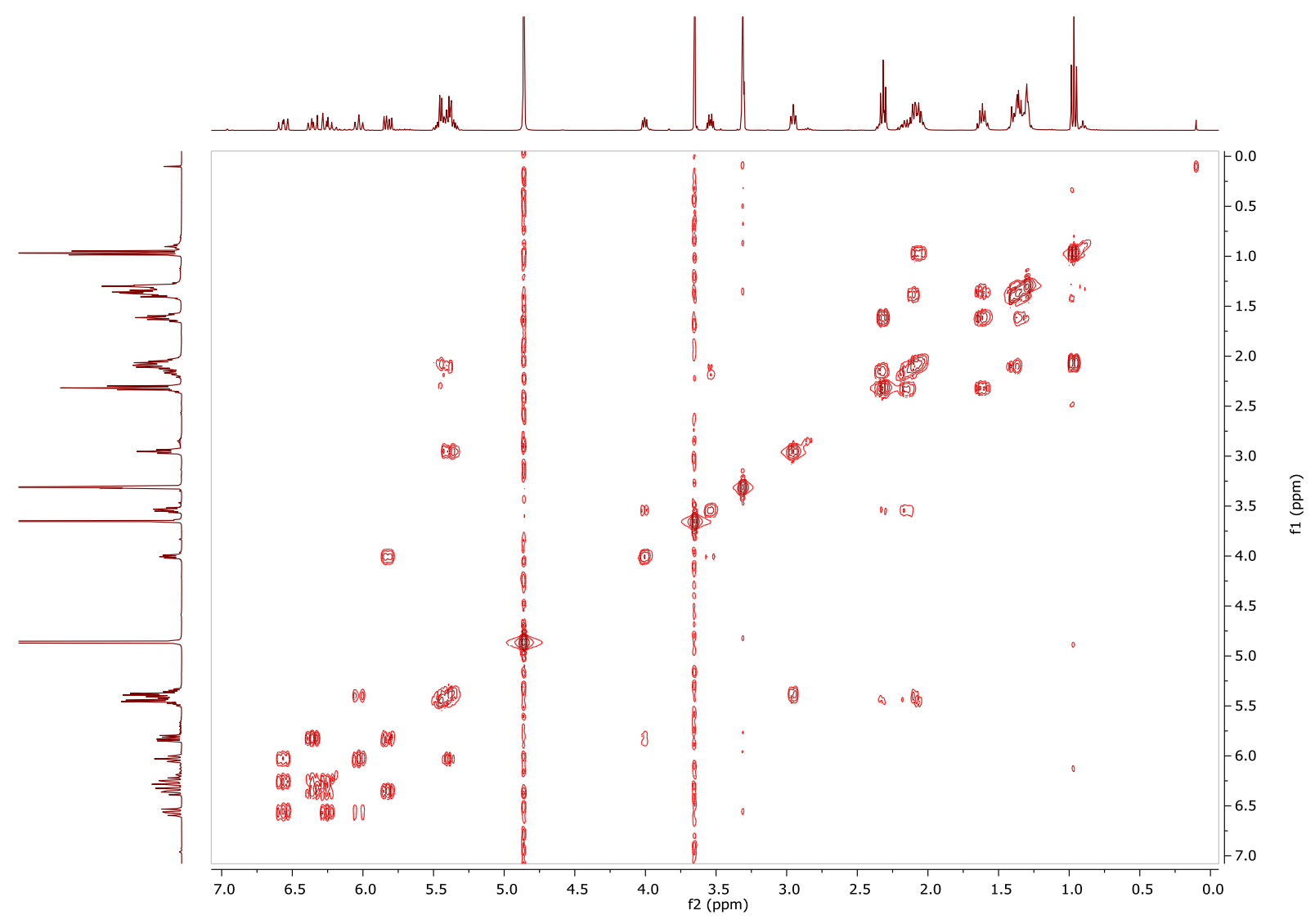

Figure S-13 COSYGP spectrum of the methyl ester of PD2 ${ }_{n-3}$ DPA $(21)$.

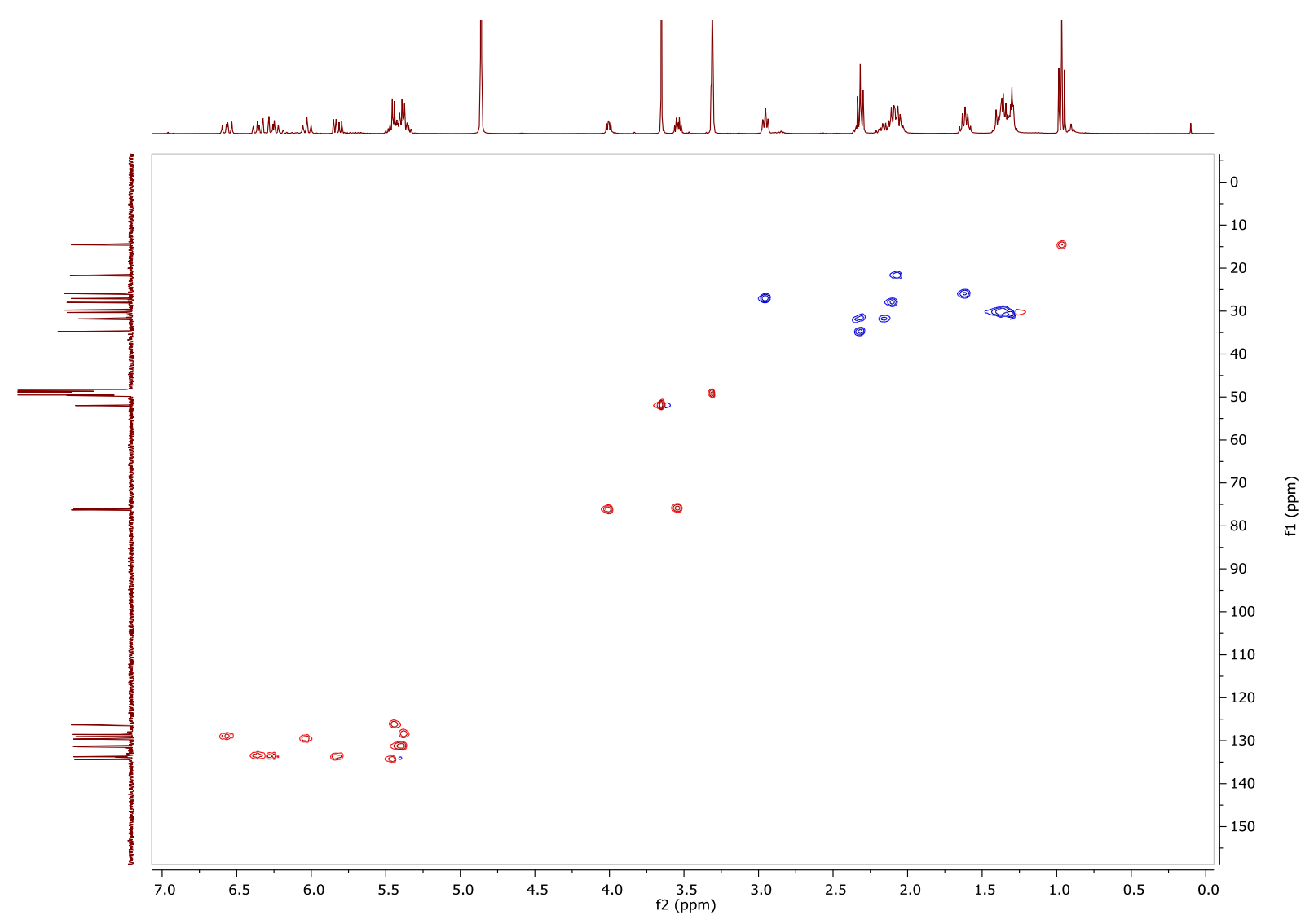

Figure S-14 HSQCEDETGP spectrum of the methyl ester of PD2 ${ }_{n-3}$ DPA $(21)$. 


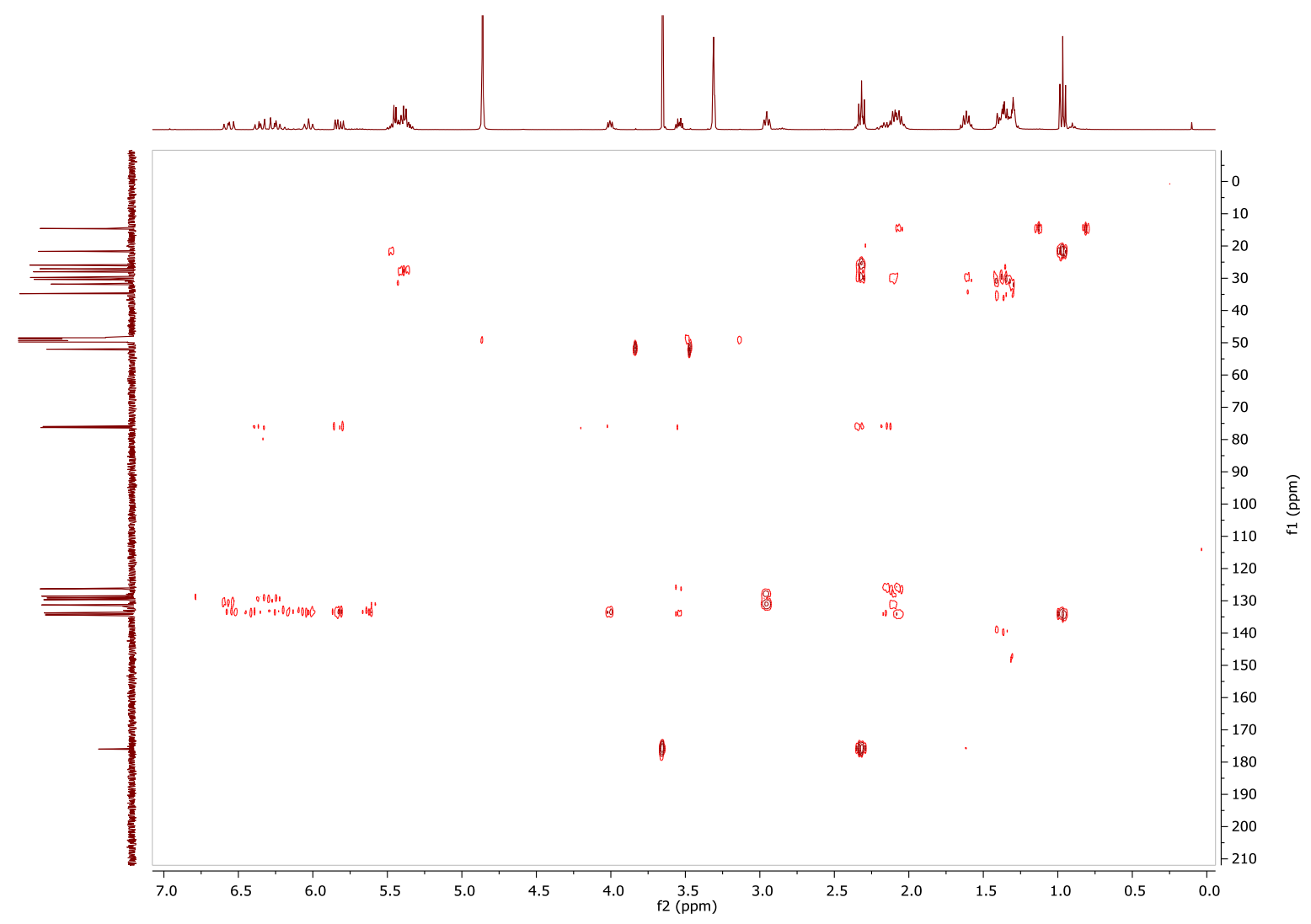

Figure S-15 HMBCGPND spectrum of the methyl ester of PD $2_{n-3}$ DPA (21).

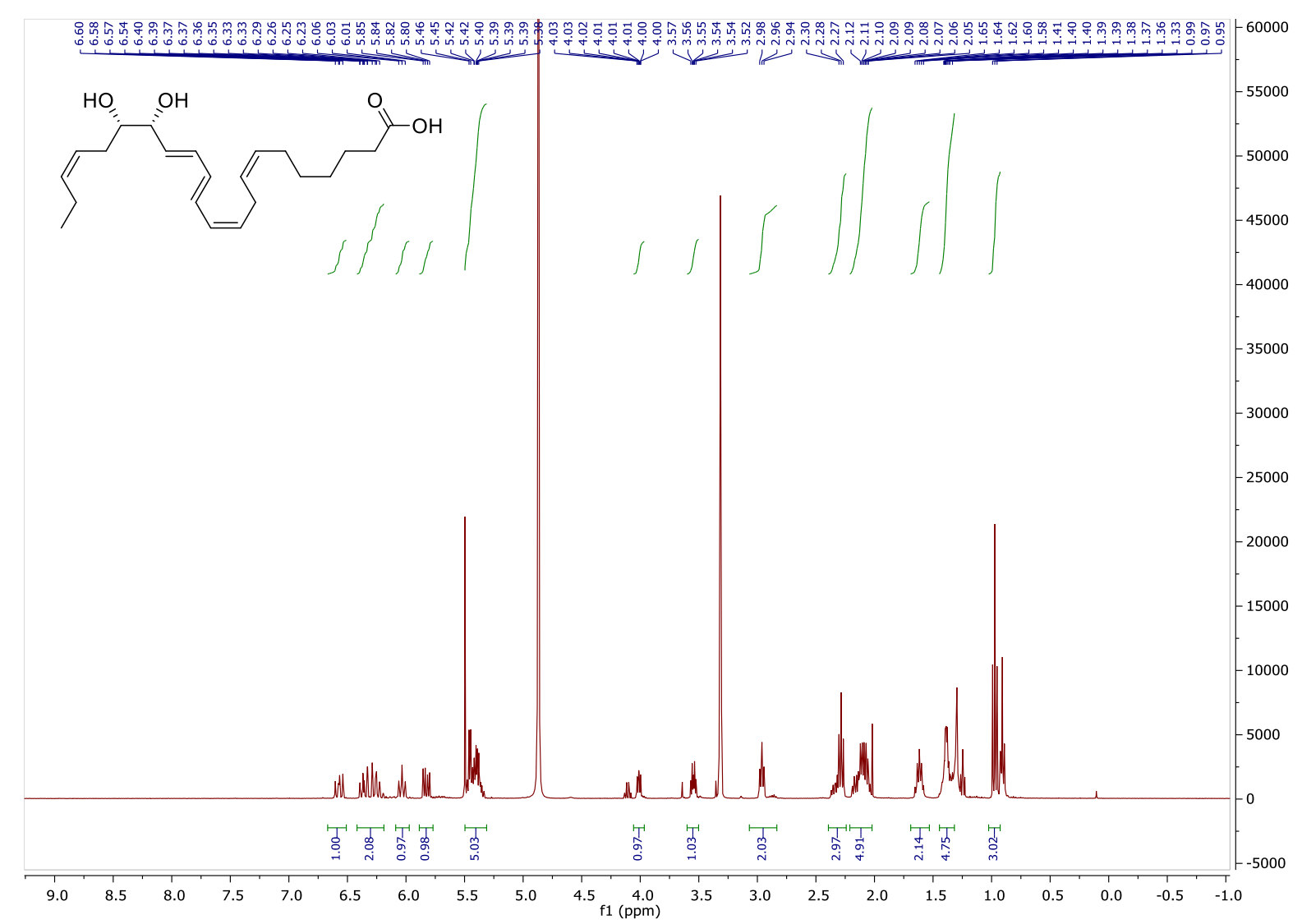

Figure S-16 ${ }^{1} \mathrm{H}-\mathrm{NMR}$ spectrum of PD2 ${ }_{\mathrm{n}-3}$ DPA $(2)$. 


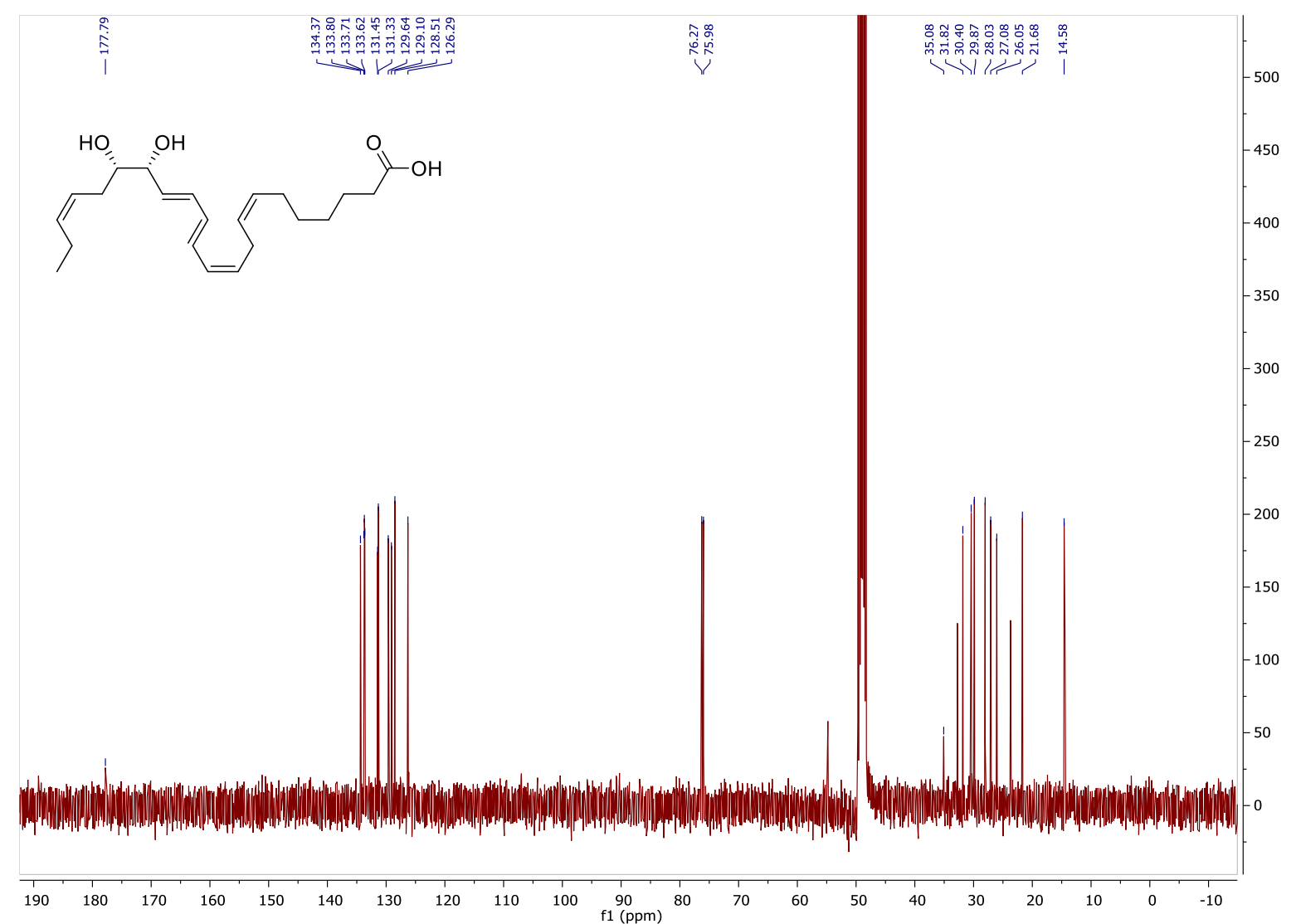

Figure S-17 ${ }^{13} \mathrm{C}-\mathrm{NMR}$ spectrum of PD2 $2_{\mathrm{n}-3 \mathrm{DPA}}(2)$.

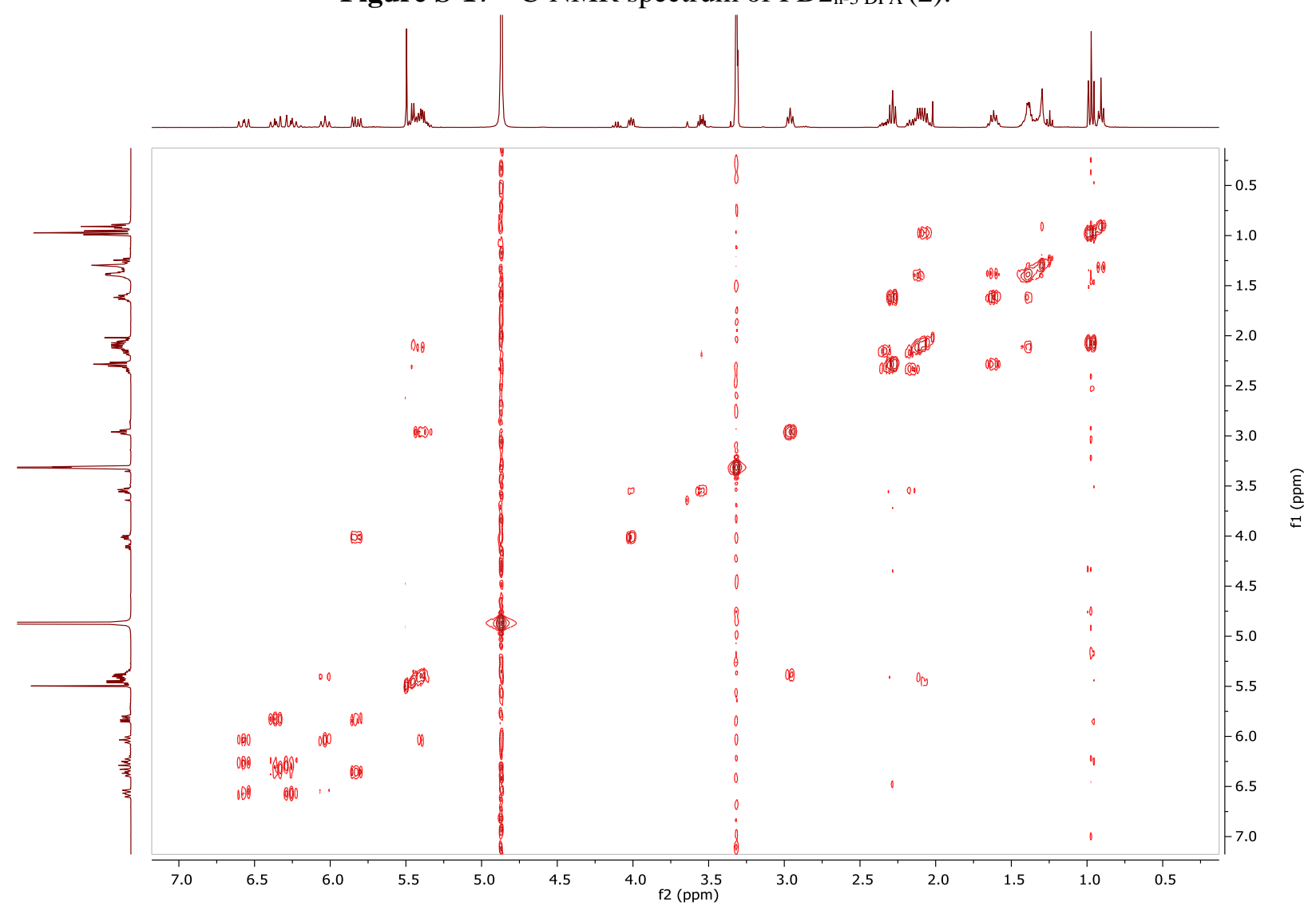

Figure S-18 COSYGP spectrum of PD $2_{\mathrm{n}-3 \mathrm{DPA}}(\mathbf{2})$. 


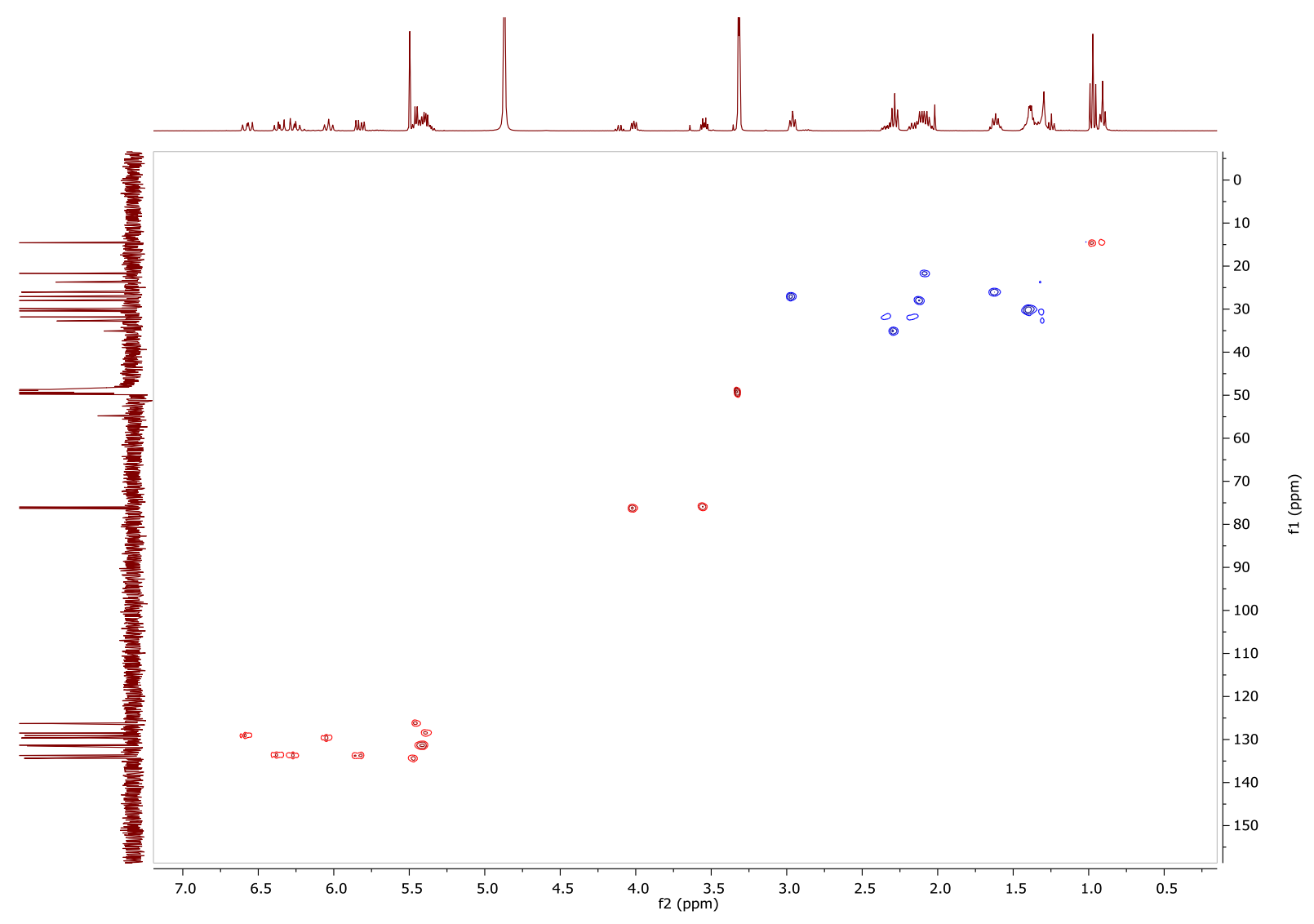

Figure S-19 HSQCEDETGP spectrum of $\operatorname{PD} 2_{n-3}$ DPA $(2)$.

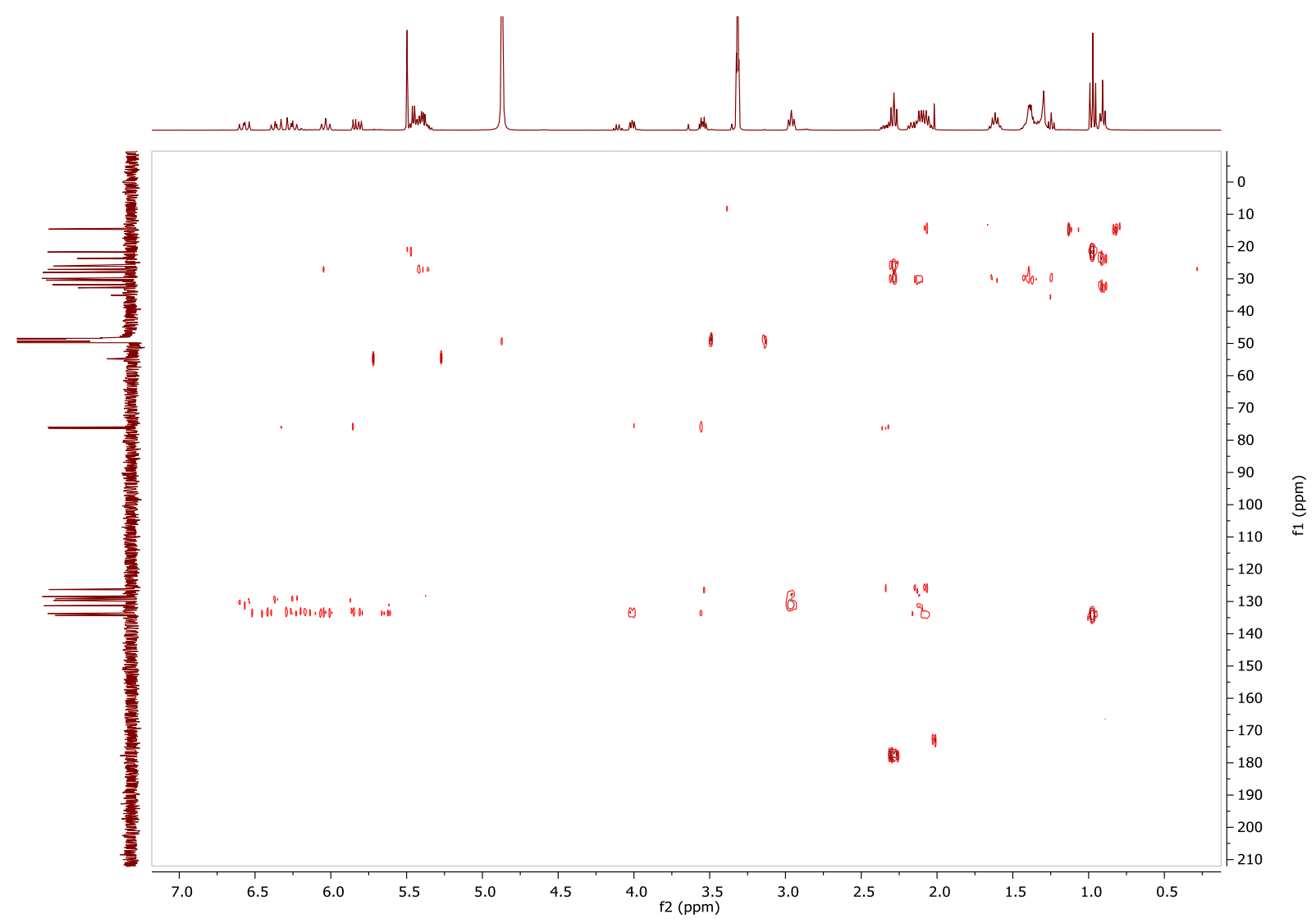

Figure S-20 HMBCGPND spectrum of PD2 $2_{\mathrm{n}-3}$ DPA $(2)$. 


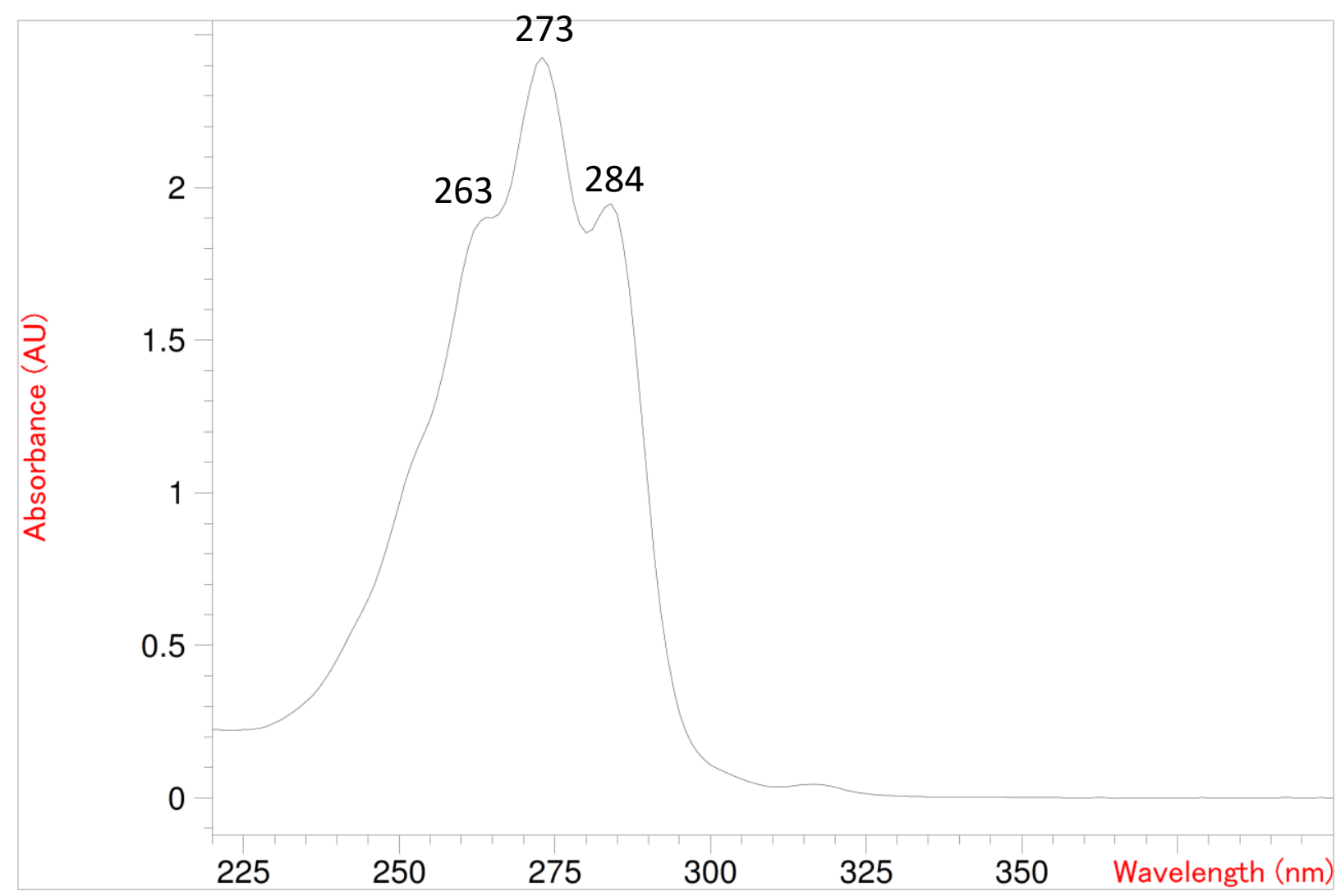

Figure S-21 UV-chromatogram of the methyl ester of PD $2_{\mathrm{n}-3 \mathrm{DPA}}(\mathbf{2 1})$.

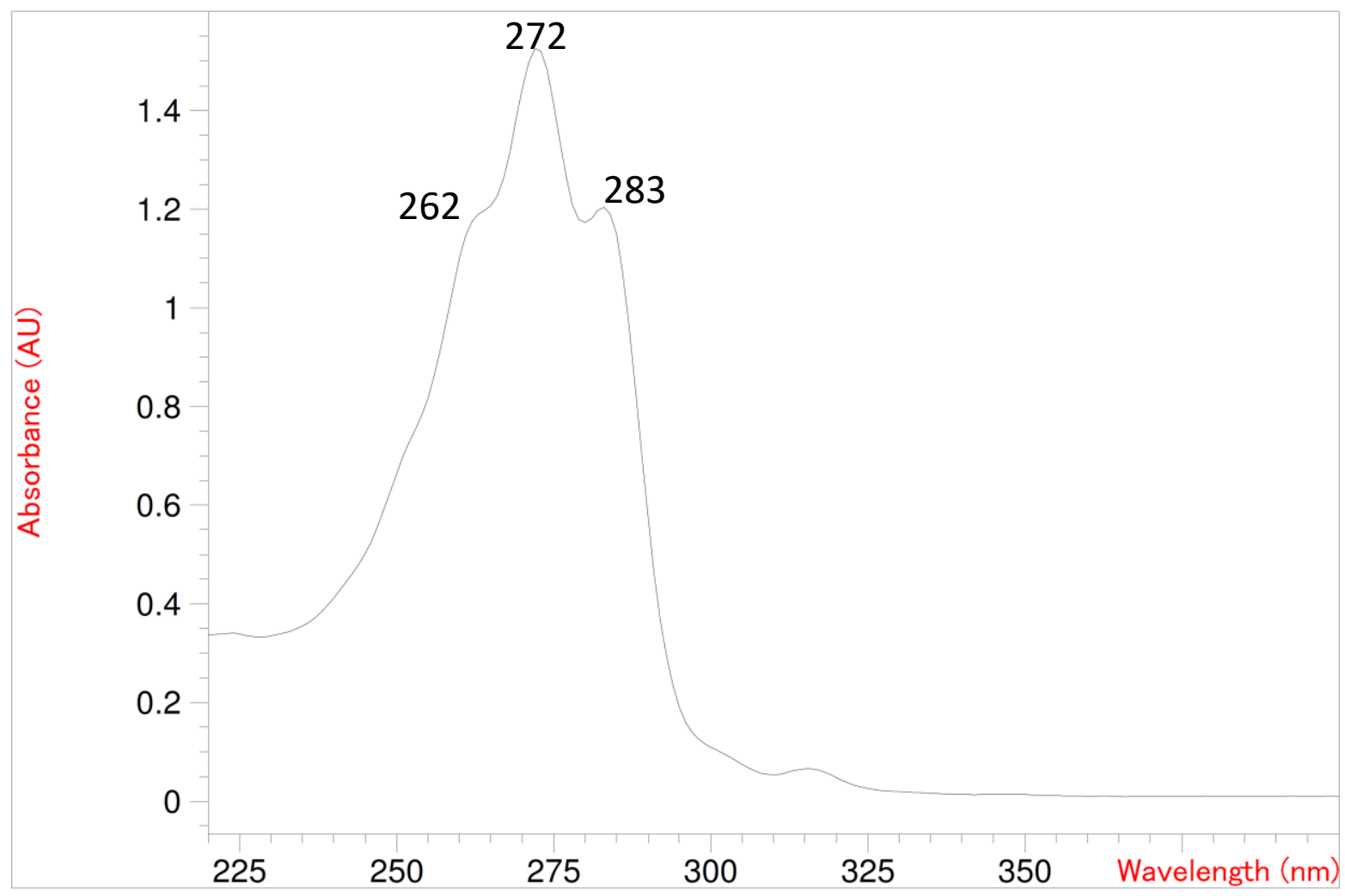

Figure S-22 UV-chromatogram of PD2 $2_{\mathrm{n}-3}$ DPA $(2)$. 


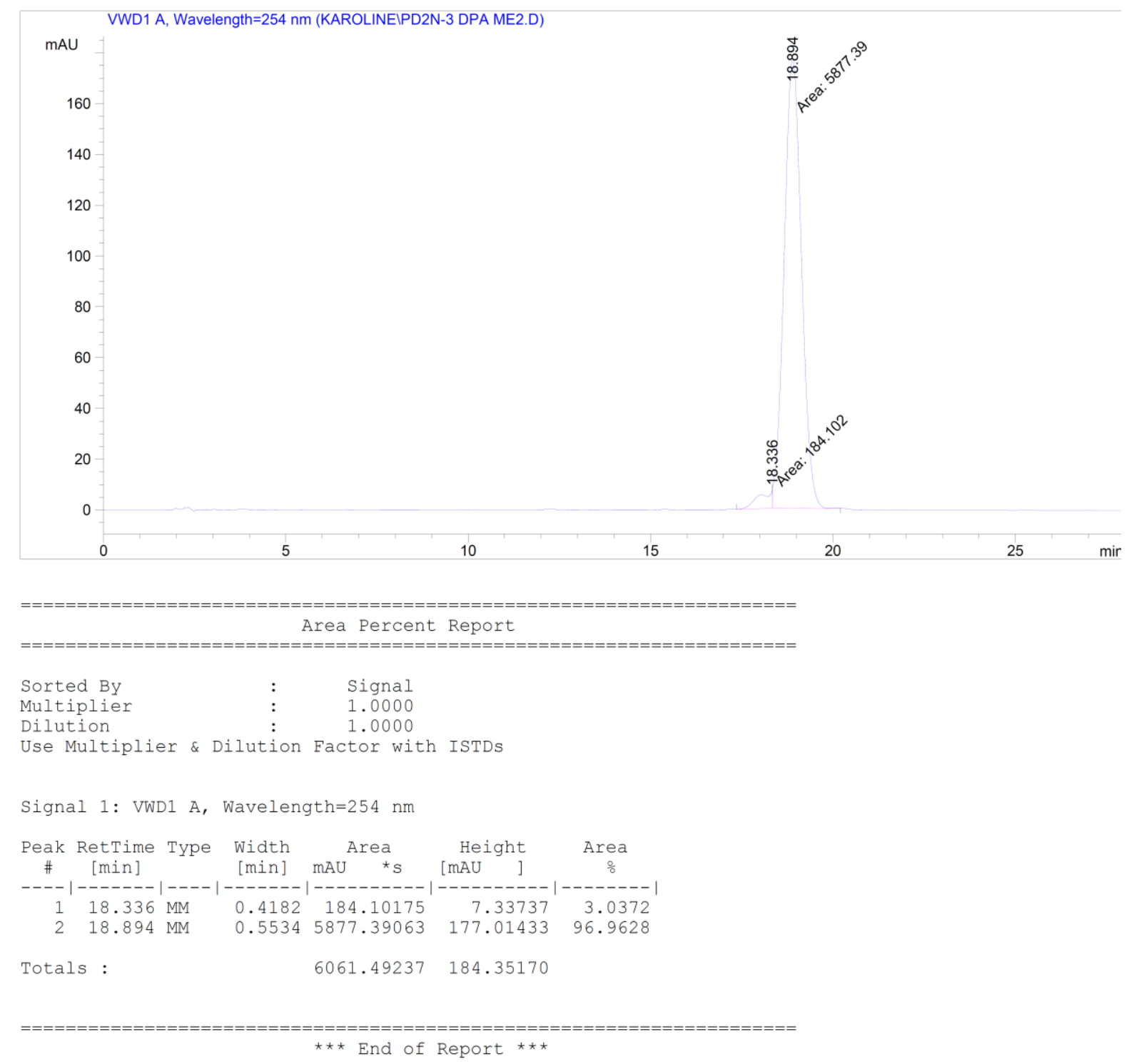

Figure S-23 HPLC-chromatogram of the methyl ester of PD2 $2_{\mathrm{n}-3}$ DPA $(\mathbf{2 1})$. 
Table S-1. Comparison of ${ }^{1} \mathrm{H}$ data of PD2 and PD2 ${ }_{\mathrm{n}-3}$ DPA (2)
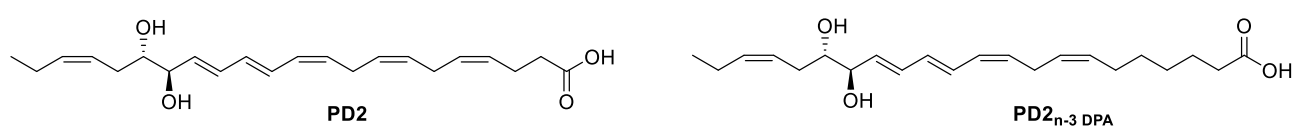

\begin{tabular}{|c|c|}
\hline PD2 & $\begin{array}{c}\text { PD2 }{ }_{\mathrm{n}-3 \mathrm{DPA}} \\
\delta_{\mathrm{H}, \text { mult., }(J \text { in Hz})^{\mathrm{a}}}\end{array}$ \\
\hline $6.7-6.5, \mathrm{dd}, 1 \mathrm{H},(14.4,11.1)$ & $6.57, \mathrm{dd}, 1 \mathrm{H},(14.3,11.2)$ \\
\hline $6.4-6.2, \mathrm{~m}, 1 \mathrm{H}$ & $6.36, \mathrm{ddd}, 1 \mathrm{H}(15.1,10.7,1.1)$ \\
\hline $6.4-6.2, \mathrm{~m}, 1 \mathrm{H}$ & $6.26, \mathrm{dd}, 1 \mathrm{H},(14.6,10.7)$ \\
\hline $6.1-6.0, \mathrm{br}, 1 \mathrm{H}(11.1)$ & $6.03, \mathrm{t}, 1 \mathrm{H}(11.1)$ \\
\hline $5.9-5.7, \mathrm{dd}, 1 \mathrm{H}(15.0,7.2)$ & $5.83, \mathrm{dd}, 1 \mathrm{H},(15.0,7.1)$ \\
\hline $5.5-5.3, \mathrm{~m}, 7 \mathrm{H}$ & $5.50-5.32, \mathrm{~m}, 5 \mathrm{H}$ \\
\hline $4.1-3.9, \mathrm{~m}, 1 \mathrm{H}$ & $4.01, \mathrm{ddd}, 1 \mathrm{H},(7.0,4.9,1.1)$ \\
\hline $3.6-3.5, \mathrm{dt}, 1 \mathrm{H},(8.1,4.8)$ & $3.55, \mathrm{dt}, 1 \mathrm{H},(8.1,4.9)$ \\
\hline $3.0-2.9, \mathrm{~m}, 2 \mathrm{H}$ & $2.96, \mathrm{t}, 2 \mathrm{H},(7.3)$ \\
\hline $2.9-2.8, \mathrm{~m}, 2 \mathrm{H}$ & - \\
\hline $2.5-2.2, \mathrm{~m}, 5 \mathrm{H}$ & $2.39-2.26, \mathrm{~m}, 3 \mathrm{H}$ \\
\hline $2.2-2.0, \mathrm{~m}, 3 \mathrm{H}$ & $2.21-2.03, \mathrm{~m}, 5 \mathrm{H}$ \\
\hline- & $1.62, \mathrm{quint}, 2 \mathrm{H},(7.3)$ \\
\hline- & $1.46-1.29, \mathrm{~m}, 4 \mathrm{H}$ \\
\hline $1.0-0.9, \mathrm{t}, 3 \mathrm{H},(7.5)$ & $0.97, \mathrm{t}, 3 \mathrm{H},(7.5)$ \\
\hline
\end{tabular}

${ }^{\mathrm{a}}$ Measured at $300 \mathrm{MHz}\left(\mathrm{CD}_{3} \mathrm{OD}\right)$. ${ }^{\mathrm{b}}$ Measured at $400 \mathrm{MHz}\left(\mathrm{CD}_{3} \mathrm{OD}\right)$. 